\title{
High-Density Lipoprotein Antioxidant Responses to High-Intensity Interval and Steady-State Moderate-Intensity Exercise
}

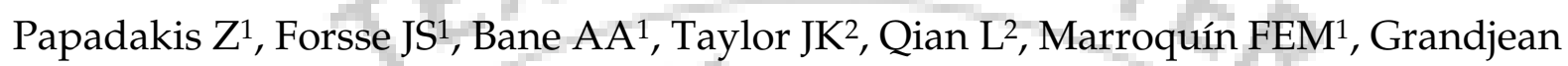
$\mathrm{PW}^{1}$

${ }^{1}$ Baylor Laboratories for Exercise Science and Technologies; Department of Health, Human Performance, and Recreation; Baylor University; Waco, TX

${ }^{2}$ Clinical Laboratory Science; Auburn University-Montgomery; Montgomery, AL Category: Doctoral

Advisor / Mentor: Grandjean, Peter W. (Peter_Grandjean@baylor.edu)

ABSTRACT

High-intensity interval exercise (HIIE) may impart health benefits beyond what is acquired through moderate-intensity steady state exercise (SSE). Paraoxonase 1 (PON1), an antioxidant associated with highdensity lipoprotein (HDL), may be altered with exercise; however, it is unclear whether HIIE provides a greater stimulus for increasing PON1 antioxidant activity than a comparable or greater amount of SSE. PURPOSE: Determine the influence of HIIE on PON1 concentration and activity relative to a comparable amount of moderate-intensity SSE and a dose that is half that of SSE. METHODS: Seventeen male participants (age $27.8 \pm 6.4 \mathrm{yr}$; weight $80.6 \pm 9.0 \mathrm{~kg}$; BMI $25.1 \pm 2.4 \mathrm{~kg} / \mathrm{m}^{2} ; \%$ fat $=19 \pm 5 ; \mathrm{VO}_{2} \max 52.1 \pm 7.5$ $\mathrm{ml} / \mathrm{kg} / \mathrm{min}$ ) underwent HIIE by treadmill running (90\% and $40 \%$ of $\mathrm{VO}_{2}$ reserve in $3: 2$ min ratio) to expend $500 \mathrm{kcals}$ (H500); HIIE to expend $250 \mathrm{kcals}$ (H250), and; SSE at 70\% $\mathrm{VO}_{2}$ reserve to expend $500 \mathrm{kcals}$ (M500) in a randomized crossover design. Intensities of all exercise conditions averaged $70 \% \mathrm{VO}_{2}$ reserve. Blood measures of total antioxidant capacity (TAC) in copper reducing equivalents, HDL ( $\square \mathrm{g} / \mathrm{mL}$ ), apolipoprotein A-1 (ApoA1: g/L), PON1 concentration (PON1c: $\square \mathrm{g} / \mathrm{mL}$ ) and arylesterase activity (PON1a: kU/L) were obtained just before, immediately after, $2 \mathrm{hr}$ and $24 \mathrm{hr}$ after exercise. Significant differences were determined using 3 by 4 repeated measures ANOVAs. Effect sizes were calculated to determine the magnitude of dependent variable responses to exercise. RESULTS: Pre-exercise HDL concentration was lower in $\mathrm{H} 250$ and increased most in $\mathrm{H} 250$ versus other exercise conditions ( $\mathrm{p}<0.001$, ES $=0.83)$. Other antioxidant responses were similar across exercise conditions. ApoA1 $(+8.0 \%)$ and PON1a $(+9.3 \%)$ increased immediately after exercise and remained elevated $24 \mathrm{hr}$ after exercise $(\mathrm{p}<$ 0.0001 for each; ApoA1 ES = 0.85, PON1a ES = 0.57). PON1c was increased $2.4 \%$ above baseline at $2 \mathrm{hr}$ post-exercise $(\mathrm{p}=0.0296, \mathrm{ES}=0.18)$ and TAC was elevated $8.6 \%$ above baseline at $24 \mathrm{hr}$ post-exercise $(\mathrm{p}=$ 0.0227 , ES $=0.48$ ). SUMMARY: HDL and HDL antioxidant properties are transiently potentiated by HIIE with as little as 250 kcals of energy expenditure. HDL antioxidant activity and total antioxidant capacity are elevated with HIIE and SSE of moderate intensity in a similar manner and are observed for up to $24 \mathrm{hr}$ after exercise. 